\title{
Pneumatosis Intestinalis following Radiation Esophagitis during Chemoradiotherapy for Lung Cancer: A Case Report
}

\author{
Noriaki Ito ${ }^{a}$ Takeshi Masuda $^{a}$ Kakuhiro Yamaguchia Shinjiro Sakamoto ${ }^{a}$ \\ Yasushi Horimasu $^{a}$ Taku Nakashima $^{a}$ Shintaro Miyamoto ${ }^{a}$ \\ Hiroshi Iwamoto $^{\mathrm{b}}$ Kazunori Fujitaka ${ }^{\mathrm{b}}$ Hironobu Hamadac \\ Noboru Hattori ${ }^{b}$ \\ aDepartment of Respiratory Medicine, Hiroshima University Hospital, Hiroshima, Japan; \\ bepartment of Molecular and Internal Medicine, Graduate School of Biomedical and \\ Health Sciences, Hiroshima University, Hiroshima, Japan; 'Department of Physical Analysis \\ and Therapeutic Sciences, Graduate School of Biomedical and Health Sciences, Hiroshima \\ University, Hiroshima, Japan
}

\section{Keywords}

Pneumatosis intestinalis · Lung cancer · Chemoradiotherapy · Chronic obstructive pulmonary disease $\cdot$ Esophagitis

\begin{abstract}
Pneumatosis intestinalis $(\mathrm{PI})$ is a rare disease that forms emphysema lesions under the mucosa and serosa of the gastrointestinal tract. We present the first case of PI following radiationinduced esophagitis during chemoradiotherapy (CRT) for lung cancer. A 74-year-old man with severe chronic obstructive pulmonary disease (COPD) was treated with CRT for lung cancer. During the treatment, he presented with vomiting and abdominal distention. CT showed pneumatosis from the esophagus to the small intestine. Severe radiation-induced esophagitis was observed, and gastrointestinal endoscopy revealed a circumferential esophageal ulcer. From these observations, this case was diagnosed as PI following severe esophagitis. A nasogastric tube was inserted, and conservative treatment with fasting, fluid replacement, and antibiotic was performed. Four days after the onset of PI, CT showed marked improvement of the pneumatosis. When CRT is performed for lung cancer patients, we should not only consider
\end{abstract}


esophagitis but also PI. The presence of COPD may be considered a specific factor for the development of severe esophagitis and the consequent $\mathrm{PI}$ in this case.

(C) 2021 The Author(s).

Published by S. Karger AG, Basel

\section{Introduction}

Pneumatosis intestinalis (PI) is a rare disease that forms emphysema lesions under the mucosa and serosa of the gastrointestinal tract, and its estimated prevalence, determined from autopsy cases, is $0.03 \%[1,2]$. PI can be divided into primary $(15 \%)$ and secondary $(85 \%)$ types [3]. Secondary-type PI occurs in patients with several conditions, such as gastrointestinal obstruction, inflammatory bowel disease, infectious enteritis, autoimmune diseases, pulmonary diseases, and the use of drugs, such as immunosuppressants and chemotherapeutic agents [4].

Three reports have shown the development of PI in patients with esophageal cancer during chemoradiotherapy (CRT) [5-7]. However, in lung cancer, there have been several reports of PI development during treatment with cytotoxic chemotherapy agents or epidermal growth factor receptor tyrosine kinase inhibitors [8, 9]. Herein, we present the first case of PI following radiation-induced esophagitis during CRT for lung cancer. In addition, this case was complicated by severe chronic obstructive pulmonary disease (COPD), and COPD is considered a specific cause of severe esophagitis resulting in the onset of PI in our case.

\section{Case Report}

A 74-year-old man was diagnosed with recurrent mediastinal lymph node metastasis of lung adenocarcinoma 3 years after surgery. He had smoked 40 cigarettes per day for 40 years (80 pack-years) and quit smoking in his late 50s. A pulmonary function test showed that the forced expiratory volume in $1 \mathrm{~s}$ was $23.5 \%$ of the predicted value, which suggested that he had GOLD stage 4 COPD. He was hospitalized and administered concurrent CRT 7 cycles of chemotherapy with weekly carboplatin $[\mathrm{AUC}=2]$ and paclitaxel $\left[35 \mathrm{mg} / \mathrm{m}^{2}\right]$ and volumetric modulated arc therapy of 66 Gy in total). Eighteen days after the start of CRT, he complained of grade 3 esophagitis symptoms according to the Common Terminology Criteria for Adverse Events version 5.0; nonsteroidal anti-inflammatory drugs and sodium alginate were administered. He also suffered from constipation due to chemotherapy and was administered magnesium oxide and sennoside. On the 32 nd day, he developed febrile neutropenia, and tazobactam/piperacillin ( $4.5 \mathrm{~g}$ intravenously every $8 \mathrm{~h}$ ), as well as daily subcutaneous injections of filgrastim $(75 \mu \mathrm{g} /$ day), was administered. Radiation therapy was suspended for 3 days. After that, radiation therapy was resumed, he vomited slightly every day from the 35th day onward, and a large amount of vomiting was observed on the 38th day. Abdominal examination showed distension and tenderness throughout the abdomen without signs of peritoneal irritation. Laboratory findings showed increased lactate levels $(11.5 \mathrm{mmol} / \mathrm{L})$ and inflammatory parameters (WBC, $12,300 / \mu \mathrm{L} ; \mathrm{CRP}, 17.21 \mathrm{mg} / \mathrm{dL}$ ), as well as decreased renal function (BUN, $46.6 \mathrm{mg} / \mathrm{dL} ; \mathrm{Cr}, 2.83 \mathrm{mg} / \mathrm{dL}$ ). In contrast-enhanced CT, extensive pneumatosis was observed from the esophagus to the stomach and upper part of the small intestine (Fig. 1). Minute gas was present in the portal vein. However, contrast-enhanced CT showed no obvious intestinal ischemia. First, a nasogastric tube was inserted for gastric decompression. A few hours later, abdominal symptoms were alleviated, and the lactate level was reduced to $2.5 \mathrm{mmol} / \mathrm{L}$.

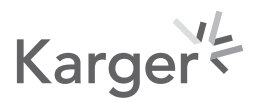



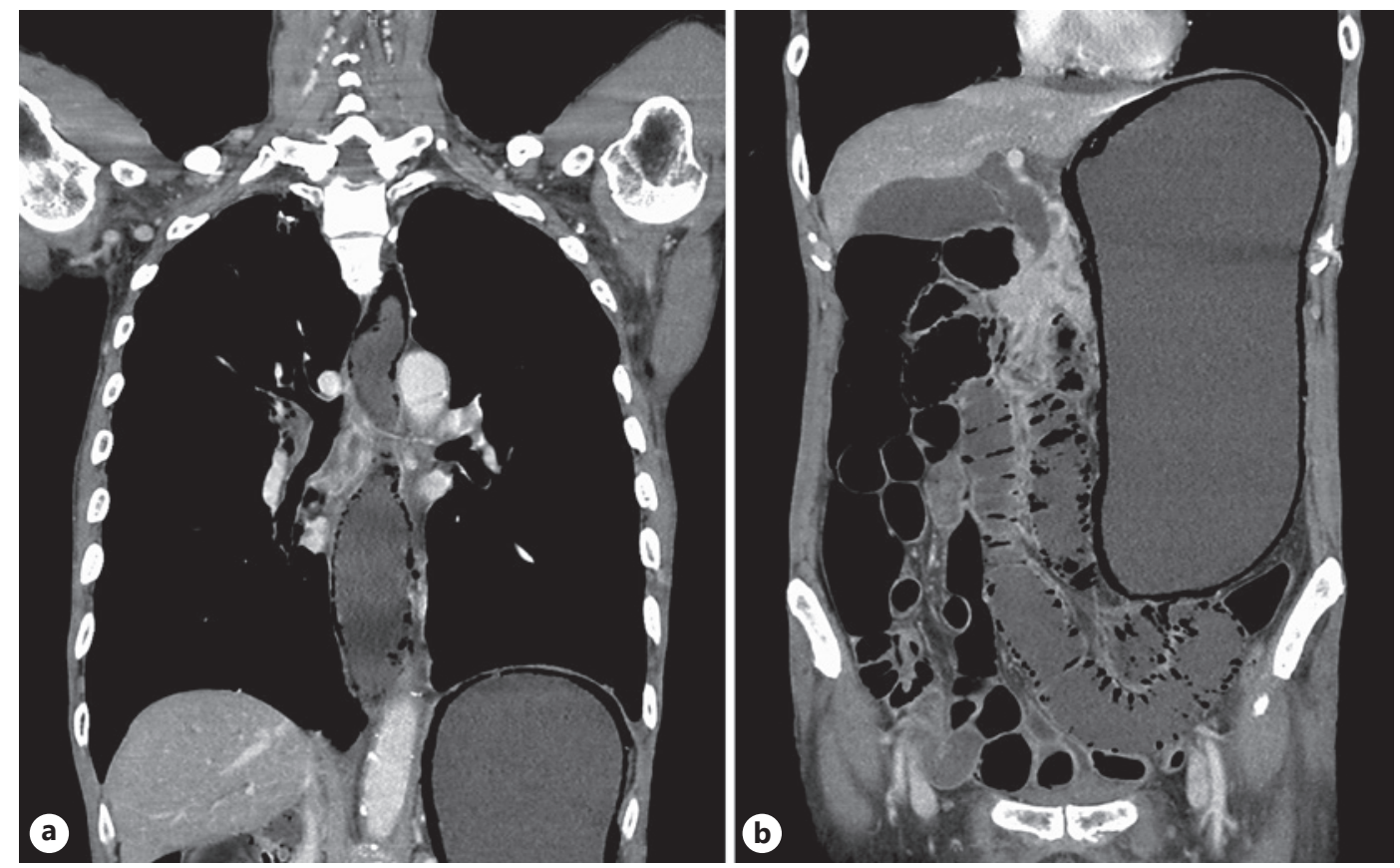

Fig. 1. Contrast-enhanced computerized tomography showing extensive pneumatosis from the esophagus to the stomach and upper part of the small intestine.

Because of this improvement, we decided that surgery was not needed and performed a conservative treatment with fasting, fluid replacement, and meropenem $1 \mathrm{~g}$ intravenously every $12 \mathrm{~h}$ ) administration in the intensive care unit. Four days after the onset of PI, CT showed marked improvement of pneumatosis, and laboratory findings also improved. Upper gastrointestinal endoscopy revealed an all-around esophageal ulcer that was covered with a white coat, and no obvious fistula was found at that time (Fig. 2). Therefore, we considered that pneumatosis occurred due to the inflow of gas into the submucosa from this esophageal lesion. After that, oral intake was resumed, and his condition improved. CRT was discontinued after 48 Gy of irradiation and 4 cycles of chemotherapy ( 7 cycles planned). After hospital discharge, he attended the hospital as an outpatient and did not relapse for PI and lung cancer.

\section{Discussion and Conclusion}

To the best of our knowledge, only 3 cases of PI during CRT for esophageal cancer have been reported in Japan [5-7]. Herein, we report the first case of PI following radiation-induced esophagitis due to CRT for mediastinal lymph node metastasis from lung cancer. Of note, severe COPD is considered a specific factor for the development of severe esophagitis and the consequent PI.

There are 2 main theories to explain the mechanism of PI onset, specifically mechanical and bacterial. The mechanical theory proposes that increased intraluminal pressure due to gastrointestinal obstruction causes mucosal rupture of the intestinal wall, resulting in the migration of gas from the gastrointestinal cavity to the intestinal wall. In contrast, the bacterial theory proposes that gas-producing bacteria, such as Clostridium difficile, enter the gastrointestinal wall and produce gas [10]. In addition, it has been reported that increased mucosal permeability due to intestinal ischemia, mucosal damage due to inflammation and chemotherapy, 

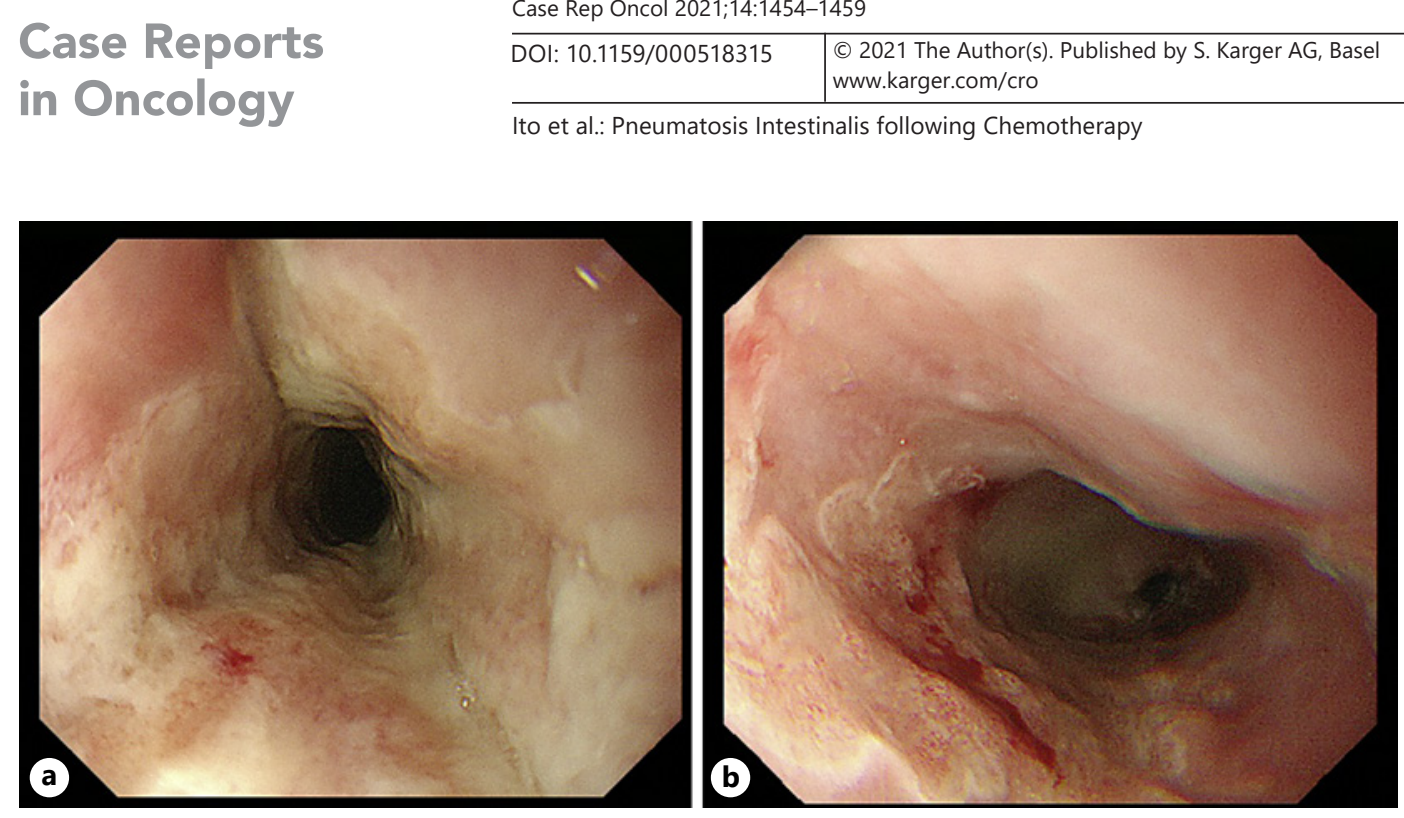

Fig. 2. Gastrointestinal endoscopy showing the all-around ulcer which was covered with a white coat (a), map-like ulcer, and friable in the esophagus (b).

and defects in the mucosal barrier caused by immunodeficiency are associated with the onset of PI [10]. In our case, pneumatosis was distributed from the esophagus to the stomach and intestinal tract, and upper gastrointestinal endoscopy showed esophageal lesions. From these observations, it was considered that the PI developed via the entry of gas from the mucosal injury of the esophagus into the submucosa, and gas spread to the stomach and intestinal tract after increased intraluminal pressure due to vomiting. Moreover, as previous reports show $[8,11,12]$, febrile neutropenia and chemotherapy would also contribute to the development of pneumatosis.

Radiation esophagitis and neutropenia are common events during CRT for lung cancer. Thus, we thought that there was a specific factor in the form of pneumatosis from the esophagus to the intestine following radiation-induced esophagitis in our case. The complication of severe COPD was thought to be involved in the development of PI. A previous review article by Boerner et al. [13] showed that $20 \%$ of 123 patients with PI had COPD. In addition, the pulmonary theory explains the mechanism of PI onset. This theory explains that the mechanism by which pulmonary disease causes PI is the gas released via the rupture of alveoli, which moves through the mediastinum and retroperitoneum into the intestinal wall [8]. However, in our case, no pneumatosis was observed in the lungs, mediastinum, or mesentery. Thus, PI in our case was not considered to have developed from the lungs. In contrast, by searching the literature that reported a relationship between COPD and damage to the intestinal mucosa, we found a report showing that smoking causes inflammation of the gastrointestinal mucosa in animals [14]. In addition, another study showed that in patients with COPD, the permeability of the intestinal mucosa is higher than that in healthy subjects [15]. This is why increased metabolic demand related to physical activity causes intestinal ischemia, resulting in intestinal mucosal damage in patients with COPD [15]. Of note, these results can also be applied to the mucous membrane of the esophagus. Based on these observations, it is considered that patients with severe COPD have a high risk of developing PI following radiation-induced esophagitis.

PI is a serious disease, with a reported fatality rate of $20-42 \%$ [10]. In previous reviews, risk factors for a lethal course of PI were reported as age $\geq 60$, signs of peritonitis, hypotension, abdominal rigidity, increased serum lactate ( $>2.0-3.0 \mathrm{mmol} / \mathrm{L})$, increased serum creatinine ( $>1.5-2.0 \mathrm{mg} / \mathrm{dL}$ ), extensive pneumatosis (including small bowel and colon), arterial or venous mesenteric occlusion, and portomesenteric venous gas [10]. Although our

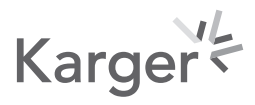


patient had many risk factors, such as old age, increased serum lactate, increased serum creatinine, widespread pneumatosis from the esophagus to the upper part of the small intestine, and portal vein gas, the patient fortunately improved with the use of conservative treatment alone. In our case, unlike the previously reported cases, the origin of pneumatosis was the esophagus. Thus, the degree of ischemia and damage to the intestinal tract were minor, and conservative treatment could only lead to a smooth improvement without surgery.

We presented the first case of PI following radiation-induced esophagitis during CRT for lung cancer in a patient with severe COPD. When CRT is performed for lung cancer patients with severe COPD, we should not only consider esophagitis but also PI.

\section{Statement of Ethics}

The study describes a case report. Therefore, it is exempt from ethics committee approval. Written informed consent was obtained from the family of the deceased patient for publication of this case report and accompanying images.

\section{Conflict of Interest Statement}

The authors have no conflicts of interest to declare.

\section{Funding Sources}

This research did not receive any specific grant from funding agencies in the public, commercial, or not-for-profit sectors.

\section{Author Contributions}

Noriaki Ito and Takeshi Masuda contributed to conceptualization. Noriaki Ito and Takeshi Masuda contributed to writing of the initial draft. Kakuhiro Yamaguchi, Shinjiro Sakamoto, Yasushi Horimasu, Taku Nakashima, Shintaro Miyamoto, Hiroshi Iwamoto, Kazunori Fujitaka, Hironobu Hamada, and Noboru Hattori contributed to review or revision. Noriaki Ito contributed to data collection. Noboru Hattori contributed to supervision.

\section{Data Availability Statement}

All data generated or analyzed during this study are included in this article. Further enquiries can be directed to the corresponding author.

\section{References}

1 Ling F, Guo D, Zhu L. Pneumatosis cystoides intestinalis: a case report and literature review. BMC Gastroenterol. 2019 Nov;19(1):176.

2 Heng Y, Schuffler MD, Haggitt RC, Rohrmann CA. Pneumatosis intestinalis: a review. Am J Gastroenterol. 1995 Oct;90(10):1747-58.

\section{Karger'}


3 Koss LG. Abdominal gas cysts (pneumatosis cystoides intestinorum hominis); an analysis with a report of a case and a critical review of the literature. AMA Arch Pathol. 1952 Jun;53(6):523-49.

4 Khalil PN, Huber-Wagner S, Ladurner R, Kleespies A, Siebeck M, Mutschler W, et al. Natural history, clinical pattern, and surgical considerations of pneumatosis intestinalis. Eur J Med Res. 2009 Jun;14(6):231-9.

5 Matsutani T, Sasajima K, Maruyama H, Miyamoto M, Yokoyama T, Suzuki S, et al. [A case of hepatic portal venous gas caused by chemo-radiation therapy for an advanced esophageal cancer]. Nihon Shokakibyo Gakkai Zasshi. 2008 Oct;105(10):1504-8.

6 Arikawa S, Uchida M, Uozumi J, Hattori C, Suzuki G, Kaida H, et al. A case of pneumatosis intestinalis with pneumomediastium occurring during chemo-radiation therapy for a esophageal cancer. Rinsho Hoshasen. 2009; 54(7):883-7.

7 Koizumi S, Miura A, Kato T, Izumi Y, Fujiwara J, Momma K. [A case of pneumatosis cystoides intestinalis receiving chemotherapy for esophageal and tonsillar cancers]. Nihon Shokakibyo Gakkai Zasshi. 2012 Dec; 109(12):2066-73.

8 Faria LD, Anjos CH, Fernandes GD, Carvalho IF. Pneumatosis intestinalis after etoposide-based chemotherapy in a patient with metastatic small cell lung cancer: successful conservative management of a rare condition. Einstein. 2016;14(3):420-2.

9 Uruga H, Moriguchi S, Takahashi Y, Ogawa K, Murase K, Mochizuki S, et al. Gefitinib successfully administered in a lung cancer patient with leptomeningeal carcinomatosis after erlotinib-induced pneumatosis intestinalis. BMC Cancer. 2018 Aug;18(1):825.

10 Torres US, Fortes CDFM, Salvadori PS, Tiferes DA, D Ippolito G. Pneumatosis from esophagus to rectum: a comprehensive review focusing on clinico-radiological differentiation between benign and life-threatening causes. Semin Ultrasound CT MR. 2018 Apr;39(2):167-82.

11 Galm 0, Fabry U, Adam G, Osieka R. Pneumatosis intestinalis following cytotoxic or immunosuppressive treatment. Digestion. 2001;64(2):128-32.

12 St Peter SD, Abbas MA, Kelly KA. The spectrum of pneumatosis intestinalis. Arch Surg. 2003 Jan;138(1):68-75.

13 Boerner RM, Fried DB, Warshauer DM, Isaacs K. Pneumatosis intestinalis. Two case reports and a retrospective review of the literature from 1985 to 1995. Dig Dis Sci. 1996 Nov;41(11):2272-85.

14 Fricker M, Goggins BJ, Mateer S, Jones B, Kim RY, Gellatly SL, et al. Chronic cigarette smoke exposure induces systemic hypoxia that drives intestinal dysfunction. JCI Insight. 2018;3(3):e94040.

15 Rutten EPA, Lenaerts K, Buurman WA, Wouters EFM. Disturbed intestinal integrity in patients with COPD: effects of activities of daily living. Chest. 2014 Feb;145(2):245-52. 